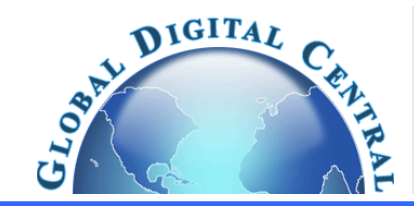

Frontiers in Heat and Mass Transfer

Available at www.ThermalFluidsCentral.org

\title{
DIRECT SIMULATIONS OF BIPHILIC-SURFACE CONDENSATION: OPTIMIZED SIZE EFFECTS
}

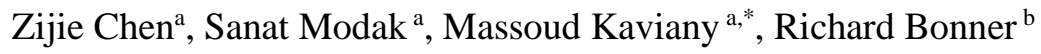 \\ ${ }^{a}$ Department of Mechanical Engineering, University of Michigan, Ann Arbor, MI, 48109, the United States \\ ${ }^{b}$ Advanced Cooling Technologies, Lancaster, PA, 17601, the United States
}

\begin{abstract}
In dropwise condensation on vertical surface, droplets grow at nucleation sites, coalesce and reach the departing diameter. In biphilic surfaces, when the hydrophobic domain is small, the maximum droplet diameter is controlled by the shortest dimension where the droplets merge at the boundary. Through direct numerical simulations this size-effect heat transfer coefficient enhancement is calculated. Then the 1-D biphilic surface is optimized considering the size-dependent hydrophilic domain partial flooding (directly simulated as a liquid rivulet and using the capillary limit), the subcooling (heat flux) and condenser length effects. The predicted performance is in good agreement with the available experiments.
\end{abstract}

Keywords: Dropwise condensation, biphilic (hydrophobic-hydrophilic) surface, size effect, partial flooding, heat transfer coefficient enhancement

\section{INTRODUCTION}

The heat transfer coefficient for dropwise condensation (DWC) on vertical surfaces is an order of magnitude larger than that for filmwise condensation (FWC) (Glicksman and Hunt, 1972), however, the DWC is challenging to maintain and easily transits to FWC (Ghosh et al., 2014). DWC consists of droplet nucleation, growth, coalescence, and departure followed by re-nucleation, with droplets ranging in size from the smallest nucleating droplets to departing droplets or even larger (Kim et al., 2015). After the droplet reaches the departure size, sweeping occurs and this could accelerate the process of dropwise condensation since the falling droplet will coalesce with and sweep the droplets in its path (Dietza et al., 2010). Under ideal dropwise condensation, the equilibrium size distribution for the droplets generally follows a power law distribution as incorporated in the so-called Rose model (Rose and Glicksman, 1972). In practice, various interfacial, constriction and other resistances can shift the droplet distribution, causing a reduction in the DWC heat transfer coefficient. To promote the DWC, micro/nano surface structures (Shang et al., 2018 and Zarei et al., 2018), as well as inorganic (gold) coatings Wilkins et al., 1973, hydrophobic porous membranes (Hu and N. Chung, 2018) and hydrophobic organic (polymers (Lu et al., 2015), self-assembled monolayers (Modak et al., 2019)), wick structure in pipe (Yunus and S. Alsoufi, 2019) have been used. To further enhance the heat transfer coefficient, bilphilic (patterned hydrophobic and hydrophilic) surfaces have been created with onedimensional (1-D) (Peng et al., 2015) and two-dimensional (2-D) (Van Dyke et al., 2015) patterns.

Due to the advantages of the DWC, its studies have a rather long history, including analytical studies of the dropwise condensation mechanism e.g., growth rate, drop size distribution, nucleation density. The direct simulations of the DWC also investigate the nucleation density, the effect of saturation temperature (Glicksman and Hunt, 1972), the drop size distribution (Leach et al., 2016), the vapor pressure effect (Wu et al., 2001), self-propelling mechanism for poly-sized droplets (Chen et al., 2019). The DWC experiments generally are for surfaces larger than several hundred microns, the experimental evaluation of the very small droplets is challenging (due to the rapid growth, large number of droplets, small scale, etc.). So far no study has addressed the surface size effect on enhancing the heat transfer coefficient of the DWC (and its direct simulations). Therefore, the surface size effect around and below hundred microns is addressed here with direct simulations using random droplet generation followed by the dropwise condensation processes. Then we consider periodic 1-D biphilic surface pattern, with the hydrophilic strips draining the condensate that formed in the hydrophobic strips by the DWC. We evaluate the surface-averaged heat transfer coefficient and compare with the available experimental results. The goal is to show the interplay of enhancement of the heat transfer by the surface size-effect of the hydrophobic domain, while avoiding flooding of the hydrophilic domain.

\section{SIZE EFFECT OF DWC}

When the domain size is below a threshold, as in biphilic patterned surfaces or partially-exposed surfaces, the droplets disappear at the boundaries. This intervening droplet removal process limits the maximum droplet size which in turn increases the heat transfer coefficient. Here we directly (numerically) simulate the DWC and evaluate the domain size effect and define the size-effect and no-sizeeffect regimes.

\subsection{Direct simulation of DWC}

\section{Heat transfer through a droplet}

Direct simulations of conduction through a droplet is made using the Star $\mathrm{CCM}+$ code. The geometry is a spherical cap, as shown in Fig. 1(a). Progressively smaller mesh size was used until heat transfer results varied by less than $1 \%$. The spherical cap can also be presented alternatively by a liquid film of uniform thickness $\delta_{l}$ (Modak et al., 2019), through

$\delta_{l}=\frac{k_{l} \Delta T_{s c}}{q}$,

\footnotetext{
*Corresponding author.Email: kaviany@umich.edu
} 
where $q$ is found from the direct simulation, $k_{l}$ is the liquid thermal conductivity $\left(0.68 \mathrm{~W} / \mathrm{m}-\mathrm{K}\right.$ at $100^{\circ} \mathrm{C}$ for water $), \Delta T_{s c}$ is the subcooling between the solid surface and liquid-vapor interface (saturated condition) and $1 \mathrm{~K}$ is used in the simulations. The ratio of $\delta_{l}$ to droplet radius $r_{d}$ is defined as $c_{k}=\delta_{l} / r_{d}$.

The heat transfer coefficient (i.e., conductance per unit area) is $G / A=h=\frac{k_{l}}{\delta_{l}}$.

The computed results for $c_{k}$ as a function of contact angle $\theta_{c}$ are given in Fig. 1(b), e.g., for $\theta_{c}=90^{\circ}, c_{k}$ is 0.109 .

\section{DWC dynamic simulations}

The software MATLAB was used to simulate the DWC under random droplet nucleation, growth, coalescence (and re-nucleation) and departure (including merging at the boundaries).

The random droplet generation is implemented with the random generator in MATLAB to generate droplets in the target area and based on the droplet nucleation density.

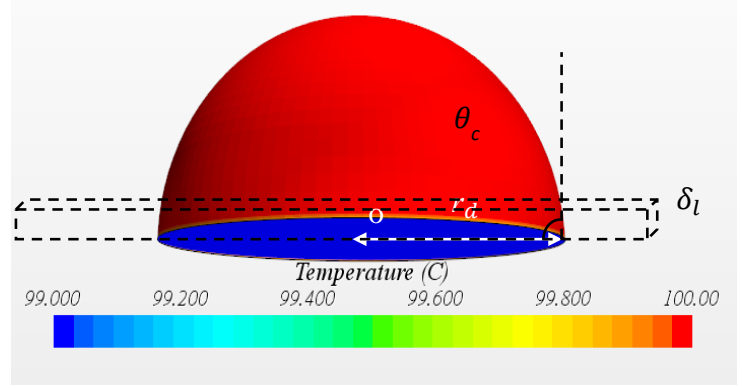

(a)

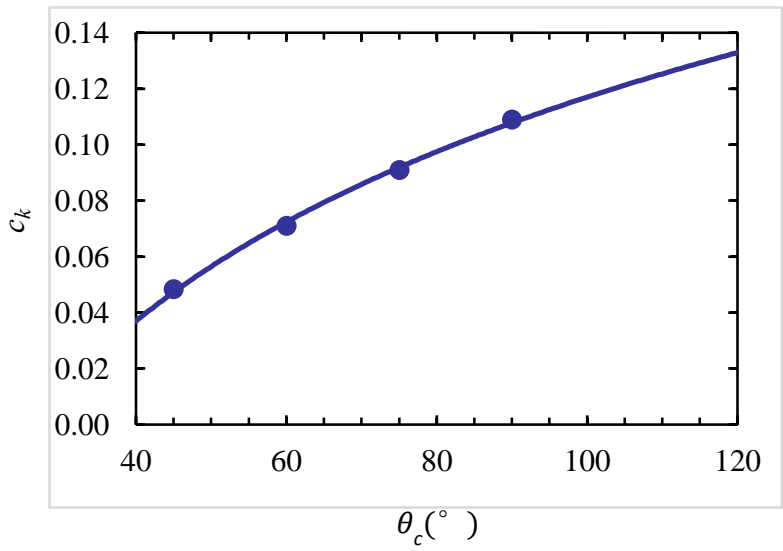

(b)

Fig. 1 (a) A snapshot of the simulated droplet temperature distribution with a contact angle of $90^{\circ}$ using the Star CCM+ code. The corresponding uniform liquid film thickness is also shown. (b) Variations of the $c_{k}$ with the contact angle $\theta_{c}$.

The volume for spherical cap droplet is given as

$V_{d}=\frac{\pi}{3} r_{d}^{3}\left(2+\cos \theta_{c}\right)\left(1-\cos \theta_{c}\right)^{2}$.

The droplet growth is by condensation of vapor on the droplet surface and by coalescence with neighboring droplets. The volumetric growth rate due to condensation is calculated from heat flow rate by

$\dot{V}_{d} \rho_{l} \Delta h_{l g}=Q$,

where $\rho_{l}$ is the liquid density for water at $100^{\circ} \mathrm{C}\left(958 \mathrm{~kg} / \mathrm{m}^{3}\right), \Delta h_{l g}$ is the heat of evaporation $(2257 \mathrm{~kJ} / \mathrm{kg})$ and
$Q=\sum \frac{k_{l} \Delta T_{s c}}{\delta_{l}} A_{d}=\sum \frac{k_{l} \Delta T_{s c}}{c_{k} r_{d}} \pi r_{d}^{2}=\pi \frac{k_{l} \Delta T_{s c}}{c_{k}} \sum r_{d}$,

$\dot{V}_{d}=\pi \frac{k_{l} \Delta T_{s c}}{c_{k} \rho_{l} \Delta h_{l g}} \sum r_{d}$.

At $k^{\text {th }}$ timestep, the first-order time discretized form of Eq. (7) gives

$V_{d_{k}}=V_{d_{k-1}}+\pi \frac{k_{l} \Delta T_{s c} r_{d}}{c_{k} \rho_{l} \Delta h_{l g}} \Delta t$

and using Eq. (4) we have

$r_{d_{k}}=\left\{V_{d_{k}} /\left[\pi\left(2+\cos \theta_{c}\right)\left(1-\cos \theta_{c}\right)^{2} / 3\right]\right\}^{1 / 3}$

The area-averaged heat flux $\langle q\rangle$ is

$\langle q\rangle=\frac{Q}{L_{z} W_{D}}$.

The thermal conductance $\langle G / A\rangle_{D}$ is

$\langle G / A\rangle_{D}=\frac{\langle q\rangle}{\Delta T_{s c}}$.

The heat flow rate for the dry surface (the surface that not covered by droplets) is negligible compared to the area covered by droplets. This is due to the very small thermal conductivity of the vapor compared to the liquid (in particular for water). Also, the radiation heat transfer from the vapor-covered surface is negligible because of the rather small subcooling common in the DWC. Due to the large nucleation density used here, the area fraction covered by droplets is also rather large. The neglecting of the heat transfer from the vapor-covered regions is also used in other direct simulations (Mikic, 1969). When two droplets touch (their center-center distance is less than the sum of their radii), a new droplet is created with the combined volume, and positioned at their center of mass. With coalescence, the combined droplet would be stored firstly, and the un-coalesced droplets would be updated at the end of each iteration. After coalescence, new droplets nucleate at the available exposed nucleation sites. Once the droplet reaches the departure radius (depending on substrate), it begins to slide along the assumed vertical surface in the direction of gravity. The sliding droplets will sweep all the droplets in their path until exiting the domain. The mechanisms of droplet departure and its speed are not addressed here, since for the optimal surface size effect, and in the case of the optimal biphilic surfaces, the droplets do not grow to be large enough to slip by gravity (even after coalescence). For larger surfaces the kinematics of the droplet departure should be addressed.

Other assumptions are: (i) before generating the random distribution for the droplets, the surface is assumed to be totally clean, (ii) all the droplets have the same initial radius $r_{i}=0.5 \mu \mathrm{m}$ (Leach et al., 2016), (iii) when a droplet touches the domain boundary, it is deleted or merged (achieved by biphilic surface) and the droplet number is reduced by one, (iv) at every time step, there may be several coalescences called the subsidiary coalescence, so it is needed to ensure that there will be no more coalescences before the next time step, (v) the average of the last several steps are used for the final distribution, (vi) the vapor pressure remains constant during condensation at the saturation temperature, for water $T_{l g}$ is $100^{\circ} \mathrm{C}$, (vii) coalescence occurs instantaneously (Rose, 1966), (viii) contact angle $\theta_{c}$ is $90^{\circ}$, and (ix) there is no wall friction.

\section{Sub-millimeter domain regime}

The droplet nucleation density $n_{o}$ is an important factor affecting the droplet number, droplet distribution, heat flux, etc. (Glicksman and Hunt, 1972). A submillimeter domain $40 \mu \mathrm{m} \times 100 \mu \mathrm{m}$, contact angle $90^{\circ}$ and variable $n_{o}$ are used to examine the predictions. With the increase in $n_{o}$, the droplet-size distribution becomes smoother, and the heat transfer coefficient rises. The droplet distributions for different $n_{o}$ are shown in Fig. 2(a), (b) and (c). The blue lines are this study and the orange straight line is the Rose model (Rose and Glicksman, 1972), i.e.,

$n_{d}\left(r_{d}\right) d r_{d}=\frac{1}{3 \pi r_{d}^{2} r_{\max }}\left(\frac{r_{d}}{r_{\max }}\right)^{-\frac{2}{3}} d r_{d}$. 


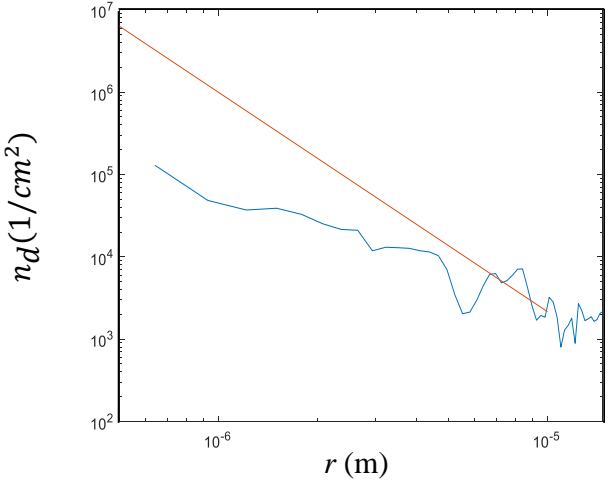

(a)

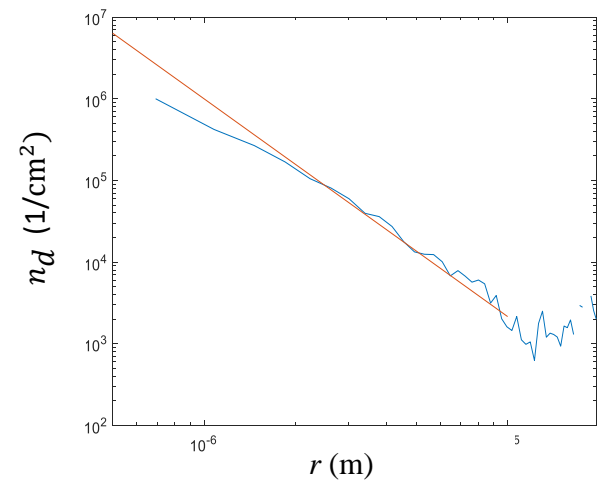

(b)

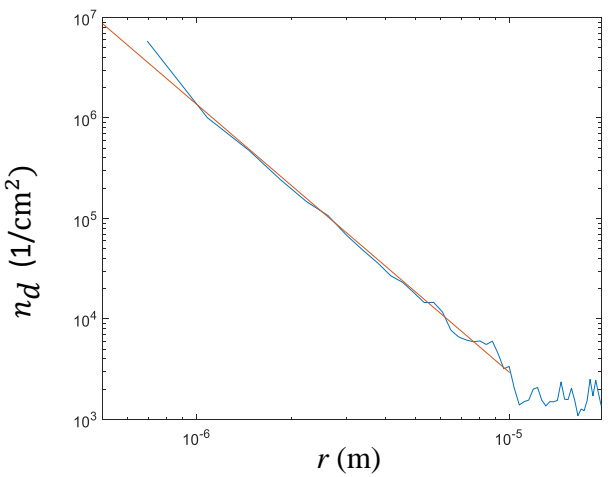

(c)

Fig. 2 Results of the droplet nucleation and growth simulation showing variations of the droplet-size number density distribution with respect to droplet radius for (a) $n_{o}=10^{6}$, (b) $10^{7}$ and (c) $10^{8} 1 / \mathrm{cm}^{2}$.

For $n_{o}=10^{8} 1 / \mathrm{cm}^{2}$, the simulations are in agreement with the Rose distribution. For $n_{o}=10^{7} 1 / \mathrm{cm}^{2}$, the results diverge for droplet radius smaller than $2 \mu \mathrm{m}$, while for $n_{o}=10^{6} 1 / \mathrm{cm}^{2}$, this occurs for smaller than $7 \mu \mathrm{m}$. These results are similar to those reported in (Glicksman and Hunt, 1972). The difference between the results with time step of $10^{-6}$ and $10^{-5}$ $\mathrm{s}$ is within $2 \%$, so $10^{-5} \mathrm{~s}$ is used.

As an example of sub-millimeter area, when the shorter dimension of the DWC surface $\left(W_{D}\right)$ is $100 \mu \mathrm{m}$, then the largest droplet radius $r_{\max }$ is assumed to be $50 \mu \mathrm{m}$. However, the largest droplet is smaller than half the width as the simulation result shows in Fig. 3(a). This is because the largest droplet may touch the boundary and be deleted before it reaches the maximum. So, the maximum droplet radius $r_{\max }$ depends strongly on the domain size. The droplet nucleation density has a relatively small influence on the $r_{\max }$. The results for $n_{o}=10^{6} 1 / \mathrm{cm}^{2}$ have some fluctuations.
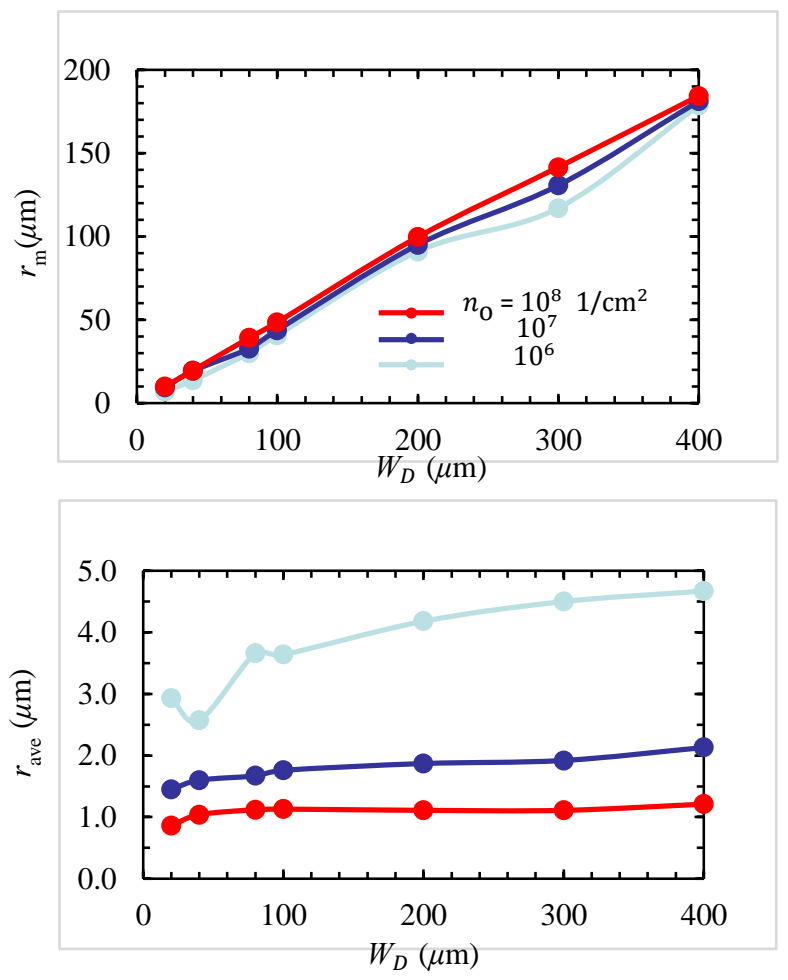

(a)

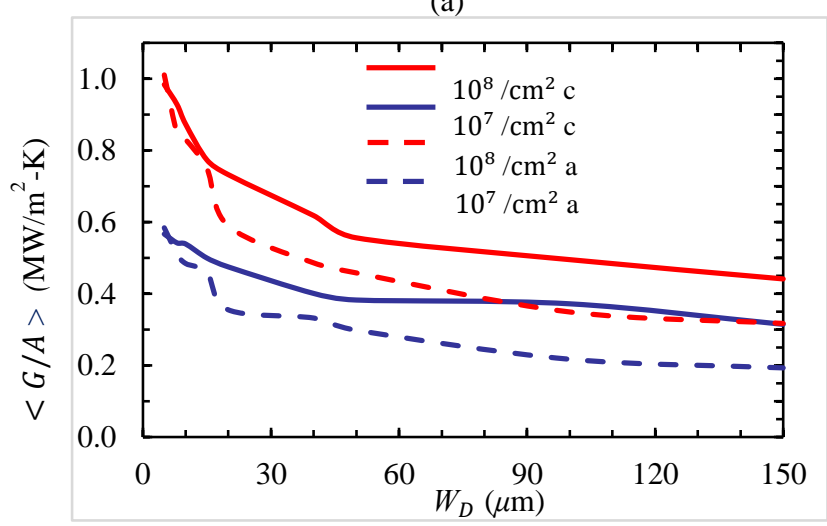

(b)

Fig. 3 (a) Variations of maximum and average droplet radius with respect to the domain width, for $n_{o}=10^{6}, 10^{7}$ and $10^{8} 1 / \mathrm{cm}^{2}$. (b) Comparison of the variations of the heat transfer coefficient using two different methods with respect to the domain width, for $n_{o}=$ $10^{7}$ and $10^{8} 1 / \mathrm{cm}^{2}$.

For $n_{o}=10^{7}$ and $10^{8} 1 / \mathrm{cm}^{2}$, the average droplet radius $r_{\mathrm{ave}}$ is nearly independent of the width as shown in. 3(a), i.e., the droplet density is large enough to counter the influence of width increase. However, for $n_{o}$ $=10^{6} 1 / \mathrm{cm}^{2}$, the $r_{\text {ave }}$ grows a bit with the width. For $W_{D}=400 \mu \mathrm{m}$ and $n_{o}$ $=10^{6} 1 / \mathrm{cm}^{2}$, the large droplet is relatively small compared to the domain width, so it does not have high chance of touching the boundary, while the nucleation density is small. It also has a little chance of coalescing with other droplets, so the large droplet more likely stays in the domain, making $r_{\text {ave }}$ relatively large compared to other $n_{o}$.

With the increase in $W_{D}$, the variations of the heat transfer coefficient (or $G / A$ ), for different $n_{o}$, are shown in Fig. 3(b). However, the extent of the deceasing trend is larger for the larger $n_{o}$. The results show that small $W_{D}$ increases the heat transfer coefficient, since the average as well as the maximum droplet radius are small, so most of area is active and the small droplets can grow at high rate.

However, using the domain size to limit the $r_{\max }$ is not accurate as Fig. 3(a) showing the $r_{\max }$ always slightly smaller than half of $W_{D}$, a "cut 
method" is proposed to simulate droplet with radius that exactly half the width we want. This is achieved by setting another domain large enough to accommodate several droplets with the maximum radius. Once the droplet reaches a preset maximum radius, it is forced to be deleted from the domain.

Compared with the heat transfer coefficient of the first method (shown with "a"), the heat transfer coefficient of the cut method (shown with "c") is higher, as shown in Fig. 3(b). This is because the former allows only for one droplet with the maximum radius and it is very unstable, the large droplets probably touch the boundary and disappear before reaching the maximum radius. With the cut method, it is certain that there are several droplets with the maximum radius, and the domain is large enough to accommodate them. Therefore, before they reach the maximum radius, they will continue to remain in the domain. With the decrease in $W_{D}$, the difference between the two methods becomes more pronounced. Since the cut method gives more accurate results, it will be used here.

\section{Millimeter domain regime}

The gravity sweeping occurs in DWC with millimeter-scale domain size. This is implemented with a sweeping mechanism by assuming that after a droplet reaches the departure size, it sweeps/shifts due to the gravity with a prescribed velocity $0.1 \mathrm{~m} / \mathrm{s}$ (Hassan, 2019). Small change about velocity will not affect the final result can be also verified. The sweeping droplets have higher chance of coalesce with other droplets within their tracts. A snapshot is also shown in Fig. 4.

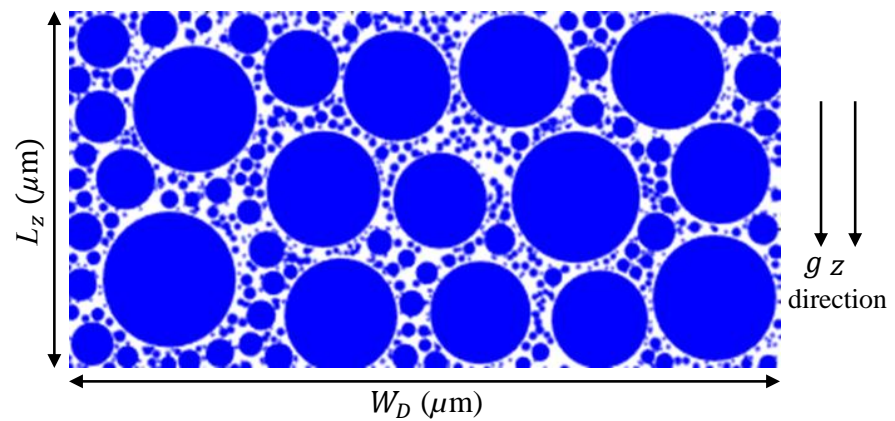

Fig. 4 Snapshot of the direct simulation of the DWC on a vertical surface. A video of the DWC dynamic simulation is available at this link site https://youtu.be/sYlTaBYhgxY. To create the video with reasonable computation, exaggerated small domain and corresponding scaled-down departing droplet radius are used.

To manage the extensive computation under very large droplet density starting with small initial radius of $0.5 \mu \mathrm{m}$, one method is ramping up the density, starting from $10^{3} 1 / \mathrm{cm}^{2}$ and progressive increasing it after getting a converging result. However, beyond $10^{6}$ $1 / \mathrm{cm}^{2}$ the computations are still extensive. Therefore, the "patch method" is proposed. This method predicts the results for high droplet nucleation density, by dividing the total simulation into two parts. One is the simulation for the large droplets starting with relatively large radius $r_{p}$ (called patch radius), where the sweeping effect is included using the departure radius of $1 \mathrm{~mm}$ for copper substrate coated with gold (Lu, et al., 2015). The other is for the small droplets starting with the initial radius of $0.5 \mu \mathrm{m}$ and ending with the $r_{p}$ (using the cut method). The final result is the sum of two parts, i.e.,

$G / A=X G_{b} / A+(1-X) G_{s} / A$,

where $X$ is the fraction of area covered by droplets with radius larger than $r_{p}$ (Le Fevfie and Rose,1966), and $X$ is

$X=1-\left(r_{p} / r_{\max }\right)^{1 / 3}$.

The results are shown in Fig. 5. The patch radii used are from 15 to $100 \mu \mathrm{m}$, for $n_{o}=10^{7}$ and $10^{8} 1 / \mathrm{cm}^{2}$. In general, there is no patch radius dependence (the difference is within $1 \%$, which is negligible). It is because that as $r_{p}$ increases, while the heat transfer coefficient of the small droplets and big droplets decrease, the fraction for small droplet increases (dominant). For large $r_{p}$, the simulation time for the large droplets is minimal for reaching the departure radius and sweeping effect, however, the simulation for the small droplets dominates the computation. The situations are reversed for small $r_{p}$. The $r_{p}=40 \mu \mathrm{m}$ is used here for computing efficiency.

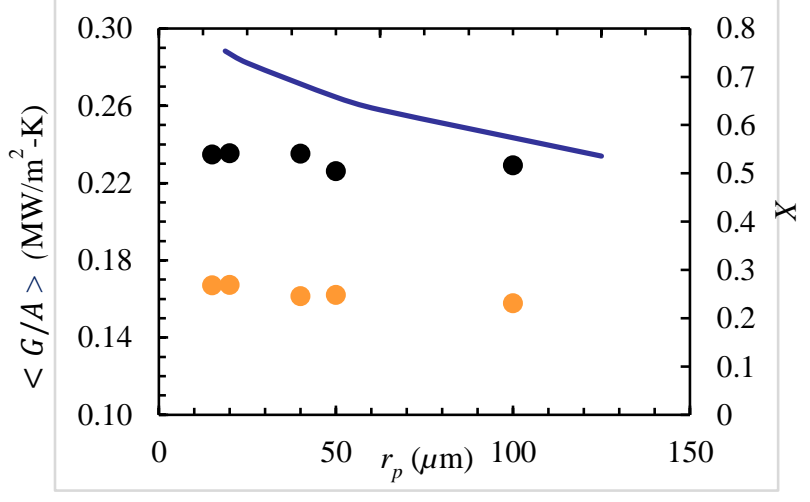

Fig. 5 Predicted variations of the thermal conductance per unit area for DWC and the fraction $X$, with respect to the patch radius. The results are for $n_{o}=10^{7}$ (orange) and $10^{8} 1 / \mathrm{cm}^{2}$ (black). The blue curve is for the $X$.

\section{Filmwise condensation}

As $W_{D}$ increases, flooding occurs in the lower portion of the condenser, i.e., the DWC will turn into filmwise condensation (FWC). The Nusselt integral analysis of the filmwise condensation on vertical surface is (Carey, 1992)

$G / A=0.943\left(\frac{k_{l}}{L_{z}}\right)\left[\frac{\rho_{l}\left(\rho_{l}-\rho_{g}\right) g \Delta h_{l g} L_{z}{ }^{3}}{k_{l} \mu_{l} \Delta T_{s c}}\right]^{1 / 4}$.

It is assumed that the flow of condensate remains laminar $\left(\rho_{g}=0.597 \mathrm{~kg} / \mathrm{m}^{3}, g=9.8 \mathrm{~m} / \mathrm{s}^{2}, \Delta h_{l g}=2257 \mathrm{~kJ} / \mathrm{kg}, k_{l}=0.679 \mathrm{~W} / \mathrm{m}-\mathrm{K}\right.$, and $\mu_{l}=2.78 \times 10^{-4} \mathrm{~N}-\mathrm{s} / \mathrm{m}^{2}$ ). For subcooling of $\Delta T_{s c}=1 \mathrm{~K}$ and $L_{z}=200$ $\mathrm{mm}$, this give $G / A=0.0174 \mathrm{MW} / \mathrm{m}^{2}-\mathrm{K}$, which is about ten times smaller than the DWC.

\subsection{Size-Effect Regime Diagram}

The cut and patch methods discussed above are used to simulate DWC with gold-coated copper substrate of variable domain size $W_{D}\left(L_{z}=W_{D}\right)$ from micrometer to meter. The conditions are: $\theta_{c}=90^{\circ}$, droplet departure radius of $1 \mathrm{~mm}$ (Lu et al., 2015), liquid-vapor interfacial conductance $(G / A)_{\lg }=5 \times 10^{6} \mathrm{MW} / \mathrm{m}^{2}-\mathrm{K}$, and the constriction resistance of $20 \%$ of the total resistance which is significant for the large droplets (Carey, 1992).

The results for the heat transfer coefficient as a function of the domain size (although using the cut method, the $x$ axis here is two times of the maximum radius) are shown in Fig. 6. There are three regimes, namely, the DWC size-effect regime, the DWC no size-effect regime (with gravity sweeping), and the FWC regime. From the FWC to no sizeeffect regime, there is an enhancement by ten folds, while from the no size-effect to the size effect regime, there is another ten folds enhancement. The band between $n_{o}=10^{7}$ and $10^{8} 1 / \mathrm{cm}^{2}$ is marked as extra enhancement although achieving $n_{o}=10^{8} 1 / \mathrm{cm}^{2}$ is rather challenging in experiments.

The results of the existing simulations (Rose, 2002) and experiments (Alwazzan et al., 2017, Hoenig, and Bonner, 2018, O'Neill, and Westwater, 1984) are also shown in Fig. 6, with good agreement with the predictions of this study. The result for condensation of a 1-D biphilic 
surface tube is adjusted for the fraction of the DWC area and only half of that area (top surface) is assumed to be not flooded. The minimum $W_{D}$ $(1 \mu \mathrm{m})$ which gives the maximum heat transfer coefficient is limited by a single droplet nucleation when there is only one droplet in the domain. So far, there are no experiments for $W_{D}<100 \mu \mathrm{m}$, so the comparison between simulations and experiments has not been extended there either.

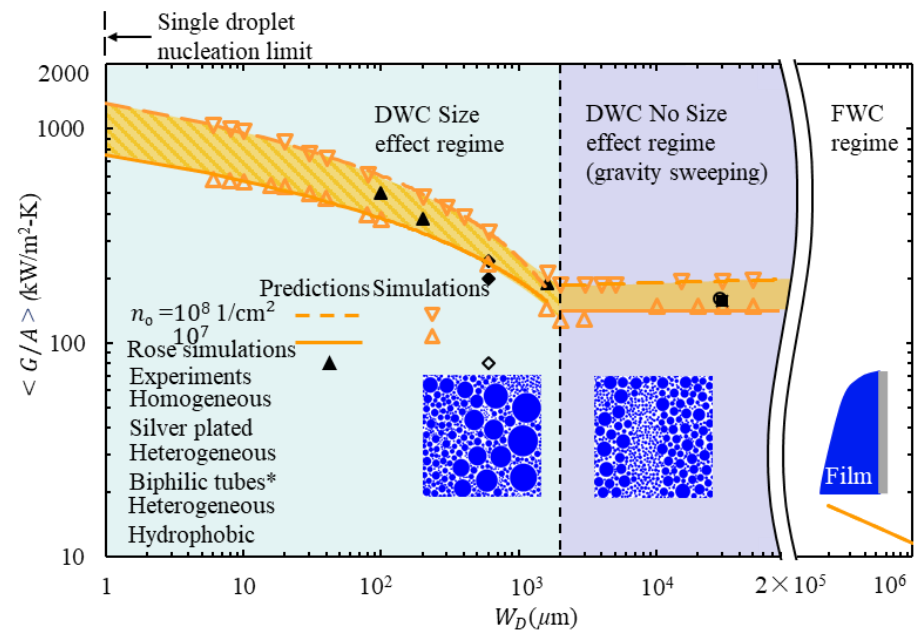

Fig. 6 The DWC size effect regime diagram showing the variations of the heat transfer coefficient with respect to the width of the hydrophobic surface. The simulation snapshots for different regimes, the experimental results and the calculated results for FWC are also shown.

\section{SIZE EFFECT in 1-D BIPHILIC SURFACE}

As the condenser length increases, the departing droplets accumulated downstream create flooding and then DWC will transition to FWC which reduces the heat transfer coefficient. What's more, the high heat transfer coefficient from size effect is based on assumption that droplets will merge at the boundaries of $W_{D}$. But this is ideal and not achievable for only hydrophobic surface in reality. To remedy this, the 1-D biphilic surface consisting of alternating hydrophobic and hydrophilic strips can help drain the condensate from hydrophobic strips into the neighboring hydrophilic strips which allow for downward flow of the liquid as isolated rivulets. This was realized in the experiment reported in (Peng et al., 2015). Here the Computational Fluid dynamics (CFD) simulations of this 1-D biphilic surface is conducted for the optimal combination of the hydrophilic strip width $\left(W_{F}\right)$ and hydrophobic strip width $\left(W_{D}\right)$ for different condenser length $\left(L_{z}\right)$ and subcooling $\left(\Delta T_{s c}\right)$.

\subsection{Direct Simulation of Liquid Rivulet}

\section{Geometry}

The schematic of the circular 1-D biphilic surface used in the experiments of (Peng et al., 2015) is shown in Fig. 7(a). It is made of unit cells of two different strips. The contact angles for hydrophobic surface and hydrophilic surface is $90^{\circ}$ and $60^{\circ}$ respectively, to match those experiments. The hydrophilic strips are considered as liquid rivulets which are assumed to have a cylindrical cap cross section formed by the surface tension. So, the maximum pressure drop along the rivulet is limited by the critical pressure across this liquid-vapor interface http://www.insula.com.au/physics/1279/L8.html. This gives

$\Delta p_{c}=\frac{\sigma}{r}=\frac{2 \sigma \sin \theta_{c}}{W_{F}}$,

where $\sigma$ is the surface tension for water at $100{ }^{\circ} \mathrm{C}, \sigma=0.059 \mathrm{~N} / \mathrm{m}, r$ is the radius of cylinder.

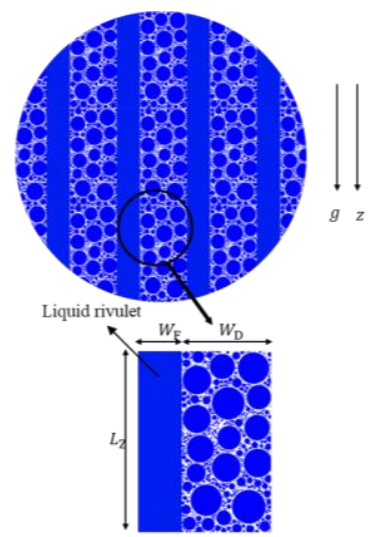

(a)

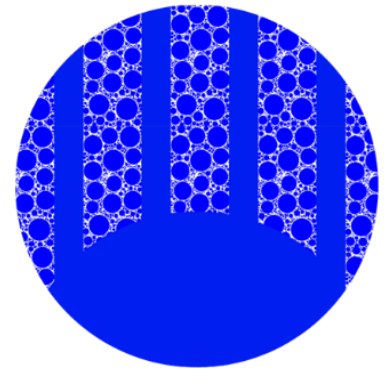

(b)

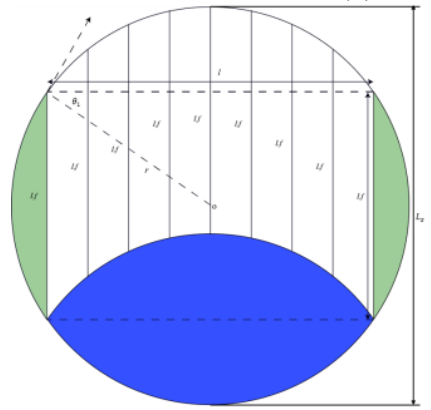

(c)

Fig. 7 Schematic of the circular 1-D biphilic surface (Peng et al., 2015), with the unit-cell strip widths and the condenser length. (b) A rendering of the hydrophilic rivulet flooding at a distance $L_{f}$ from the top, results in the partial flooding of the lower part of the condenser. (c) Details in geometry plot of the flooded lower region and its geometric parameters.

\section{Flooding}

The condensate formed within $W_{D}$ is drained along the $z$ direction (along the gravity vector) in this liquid rivulet. If rivulet viscous pressure drop $\Delta p$ that getting from the CFD simulation is larger than $\Delta p_{c}$, there is flooding downstream along $L_{z}$. A location is marked as $L_{f}$ which is defined as critical length (where $\Delta p=\Delta p_{c}$ ). For $L_{z}>L_{f}$, it is assumed that the surface floods so that region below becomes ineffective area with negligible heat transfer, as shown in Fig. 7(b). The geometric relation for the 1-D biphilic circular surface and the related parameters are shown in Fig. 7(c). The blue segment is flooded area and the rest is effective area. The radius $r$ is the half of the total length $L_{z}$. The parameters $l$ and $\theta_{1}$ shown in Fig. 7(c) are calculated by

$l=2\left[r^{2}-\left(\frac{L_{f}}{2}\right)\right]^{20.5}$,

$\theta_{1}=\arccos \left(\frac{0.5 l}{r}\right)$

The areas of the white-marked region " $w h$ " and the green region " $g r$ " in the Fig. 7(c) are

$A_{w h}=L_{f} l$,

$A_{g r}=2 A_{\text {arch }}=2\left(\pi r^{2} \frac{2 \theta_{1}}{2 \pi}-0.5 L_{f} 0.5 l\right)=2\left(r^{2} \theta_{1}-0.25 L_{f} l\right)$.

The total effective area is the sum of white and green parts, $A_{e}=A_{w h}+A_{g r}=L_{f} l-2\left(r^{2} \theta_{1}-0.25 L_{f} l\right)$.

The DWC width fraction is

$\alpha_{1}=\frac{W_{D}}{W_{D}+W_{F}}$.

The area fraction for effective area with partial flooding is 
$\alpha_{2}=\frac{A_{e}}{A}=\frac{L_{f} l-2\left(r^{2} \theta_{1}-0.25 L_{f} l\right)}{\pi r^{2}}$.

The heat flux for the 1-D biphilic surface under partial flooding is $q=<G / A>{ }_{D} \Delta T_{s c} \alpha_{1} \alpha_{2}$,

where $\langle G / A\rangle_{D}$ is thermal conductance from Fig. 6 with size effect of DWC and $n_{o}=10^{7} 1 / \mathrm{cm}^{2}$.

The thermal conductance for the 1-D biphilic surface is

$<G / A>=<G / A>_{D} \alpha_{1} \alpha_{2}$.

The total heat flow rate is

$Q_{D}=<G / A>_{D} \Delta T_{s c} W_{D} L_{z}=Q_{F}=\dot{m} \Delta h_{l g} W_{F} L_{z}$.

The mass flux is given by

$\dot{m}=\frac{W_{D}}{W_{F}} \frac{<G / A>_{D} \Delta T_{s c}}{\Delta h_{l g}}$.

The Star CCM+ code is used for the calculation of the rivulet viscous flow under gravity. Typical predicted pressure drop profile, pressure distribution along the rivulet with the marking of the critical pressure drop (which indicates flooding beyond this distance $L_{f}$ ) and the liquid velocity vector field are shown in Figs. 8(a), (b) and Fig. 9. Fig. 8 and Fig. 9 are all for $W_{F}=400 \mu \mathrm{m}$ and $L_{z}=25 \mathrm{~mm}$. The initial condition for the liquid rivulet simulation is $p=0 \mathrm{~Pa} ; u_{x}=0 \mathrm{~m} / \mathrm{s}, u_{y}=0 \mathrm{~m} / \mathrm{s}, u_{z}=0$ $\mathrm{m} / \mathrm{s}$. The boundary condition are: (i) the top curved surface is wall type (slip surface with zero shear stress), (ii) the bottom surface is mass flow inlet type (no slip with specific mass flux given), (iii) the outlet surface is constant pressure outlet type ( $p=0 \mathrm{~Pa}$ ), and (iv) the inlet arch surface is wall type (no slip surface).

In Figs. 8(a), (b), the liquid flows along the direction of gravity $(z$ direction) and the total pressure drop is $482 \mathrm{~Pa}$. The maximum pressure appears at the entrance, and the minimum pressure is $0 \mathrm{~Pa}$ set at the end. The maximum velocity is at the outlet, which is $0.233 \mathrm{~m} / \mathrm{s}$. A close-up of the liquid velocity vector field within the rivulet near the exit region is also shown in Fig. 9.

To compare the predictions of the $W_{D}$ size effect and flooding in $W_{F}$, the experimental conditions (Peng et al., 2015) of $W_{D}=950 \mu \mathrm{m}$, and variable $W_{F}$ are imposed. The pressure drop within $W_{F}$ as a function of $W_{F}$ with circular condenser of diameter $L_{z}$ are shown in Fig. 10(a) and for subcooling of 2,4 , and $6 \mathrm{~K}$. The corresponding critical pressure drop for the given $W_{D}$ is also marked. (a)

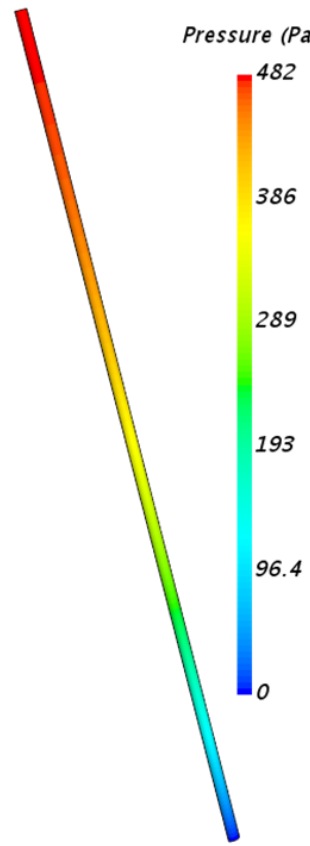

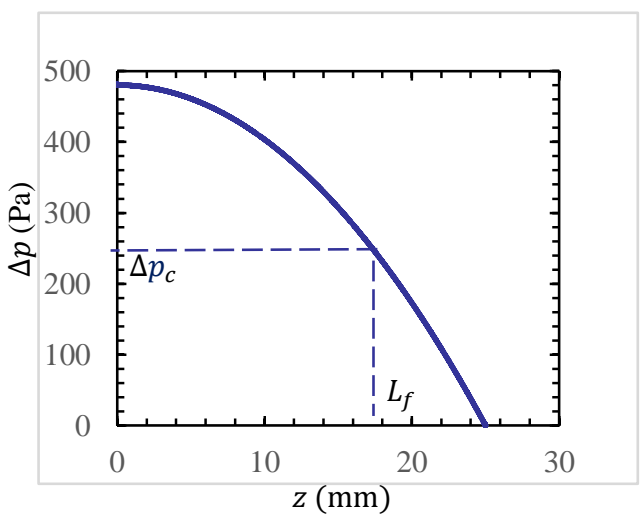

(b)
Fig. 8 (a) Predicted pressure profile along the liquid rivulet flowing down the hydrophilic strip (b) Pressure distribution along the rivulet with respect to distance from origin in $z$ direction.

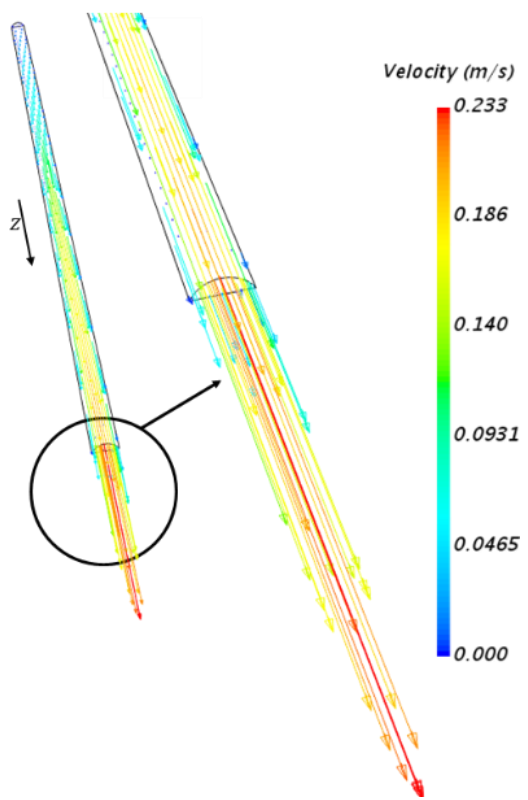

Fig. 9 Predicted liquid velocity vector field within the rivulet and the close-up near the exit region. The results are all for $W_{F}=400 \mu \mathrm{m}$ and $L_{z}=25 \mathrm{~mm}$.

With increase in $W_{F}$, the rivulet pressure drop decreases, which is duo to viscosity, the critical pressure drop also decreases but not as much as viscous pressure drop. Therefore, the intersections indicate the point that flooding starts.

Figure 10(b) shows the variations of the flooding length $L_{f}$ with respect to $W_{F}$, for three different subcooling. These are the $\mathrm{x}$ axis value for intersection points between the critical pressure horizontal line and the curve in Fig. 8(b). And there is a critical hydrophilic strip width $W_{F, c}$ where $\Delta p=\Delta p_{c}\left(L_{z}=L_{f}\right)$. For $L_{z}>L_{f}$, the downstream of the $L_{f}$ will flood as rendered in Figs. 7(b) and (c). With the decrease in $W_{F}, L_{f}$ decreases. With a larger subcooling, $L_{f}$ decreases since the condensate mass flux entering $W_{F}$ increases.

From the above results, the variations of the $W_{F, c}$ with respect to the $W_{D}$ is shown in Fig. 11. Showing that for given $W_{D}$, there is an optimal combination of two strip widths for subcooling of $0.1,0.5,1,2,4$, and 6 $\mathrm{K}$. The larger the subcooling, the larger is $W_{F, c}$. With a decrease in $W_{D}$, the $W_{F, c}$ also decreases. However, the drop is not as pronounced as that in the $W_{D}$. As $W_{D}$ tends to zero, all results tend to converge to zero.

\subsection{Results and Comparison with Experiment}

From Fig. 10(b) and Eqs. (17)-(27), the results for variations of the surface averaged heat flux and heat transfer coefficient with respect to the $W_{F}$ are shown in Figs. 12(a) and (b), for three subcooling and given $W_{D}$ and $L_{z}$, for the circular condenser of Fig. 7(a). The area-averaged heat flux first increases with $W_{F}$ due to an increased condensate draining capacity and then decreases owing to a smaller area fraction (occupied by the DWC strip) available for heat transfer. The predictions are in good agreement with the experimental results of (Peng et al., 2015). The shaded region indicates partial flooding when the $W_{F}$ is smaller than the $W_{F, c}$, which are 495,634 , and $738 \mu \mathrm{m}$ for subcooling of 2,4 , and $6 \mathrm{~K}$ respectively. The dashed line indicates the ideal heat flux under no flooding (extremely small subcooling). The average heat flux is proportional to the subcooling, while the average heat transfer coefficient is independent of subcooling when there is no flooding. Upon flooding, the heat transfer coefficient suffers depending on subcooling.

Figures 12(c) and (d) show similar variations, but with respect to the $W_{D}$ while $W_{F}$ is constant. With increase in the $W_{D}$, the heat flux increases, reaching a maximum and then decreases. So, for a given $W_{F}$ the hydrophobic strip cannot be too small (not enough heat transfer area) or 


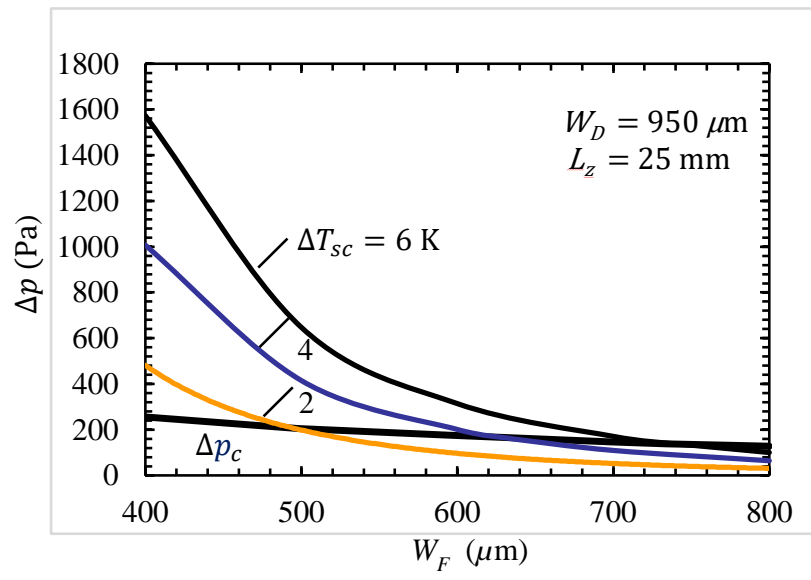

(a)

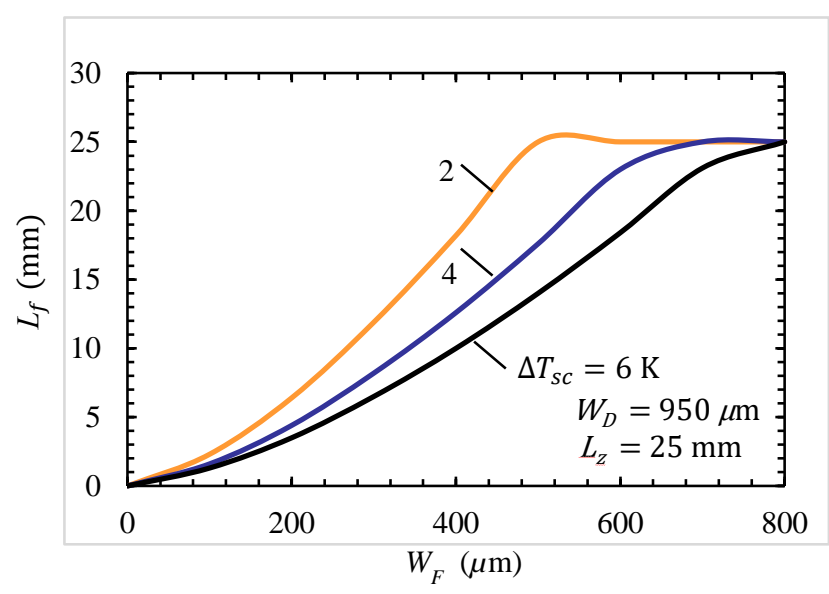

(b)

Fig. 10 (a) Variations of the rivulet pressure drop with respect to the hydrophilic strip width of the 1-D biphilic surface, for subcooling of 2,4 , and $6 \mathrm{~K}$. The variation of the capillary pressure with respect to the hydrophilic strip width is also shown. (b) The variations of the flooding length with respect to the hydrophilic strip width, for subcooling of 2,4 , and $6 \mathrm{~K}$.

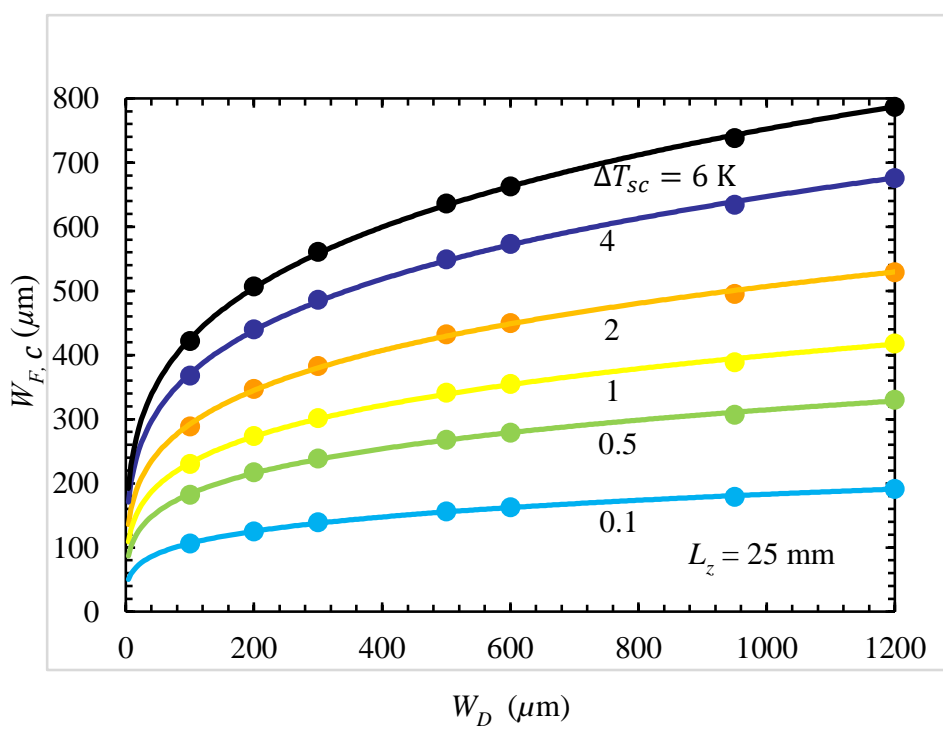

Fig. 11 Variations of the critical hydrophilic strip width (i.e., when flooding starts to occur) with respect to the hydrophobic strip width, for subcooling of $0.1,0.5,1,2,4$, and $6 \mathrm{~K}$.

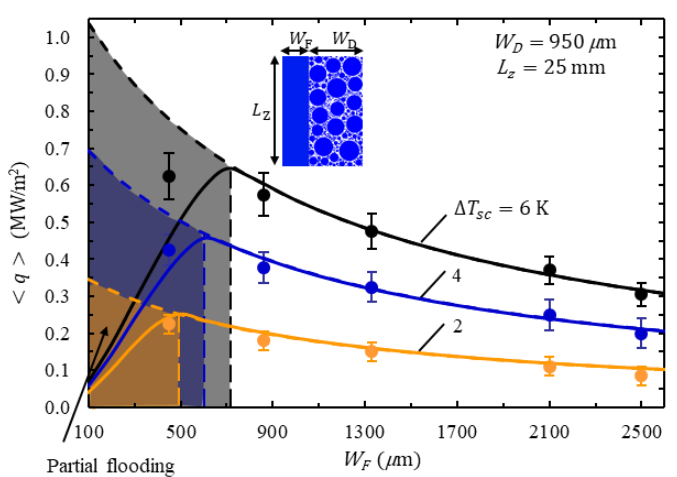

(a)

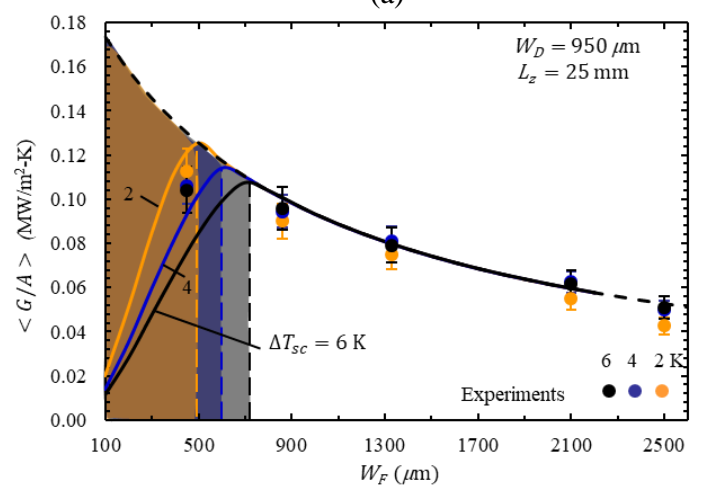

(b)

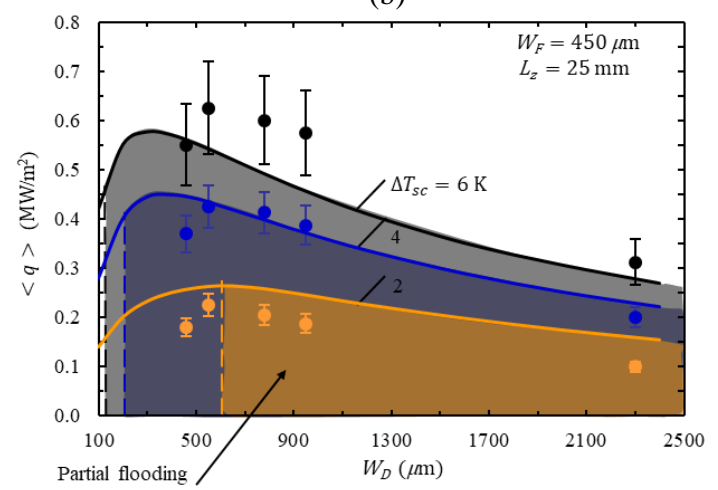

(c)

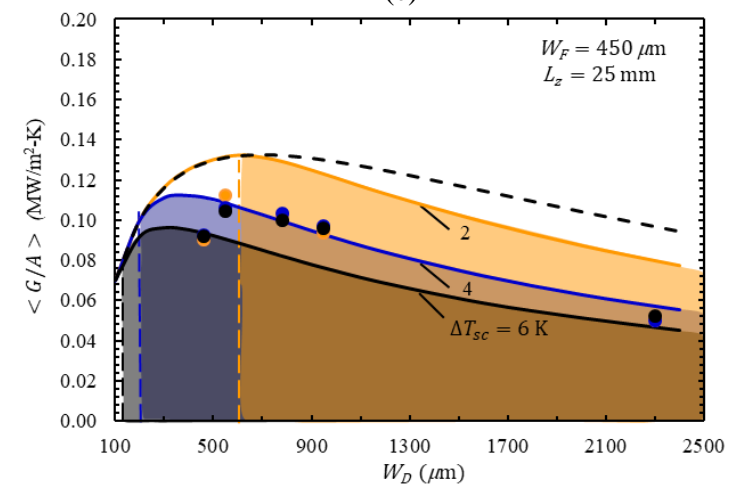

(d)

Fig. 12 Variations of the predicted (a) heat flux and (b) heat transfer coefficient with respect to the hydrophilic strip width (for hydrophobic strip width of $950 \mu \mathrm{m}$ ); Variation of the predicted (c) heat flux and (d) heat transfer coefficient with respect to the hydrophobic strip width (for hydrophilic strip width of $450 \mu \mathrm{m}$ ) for different subcooling, and comparison with the experimental results. The regimes of partially flooding are marked with shades. For (d) the predicted limiting case of zero subcooling is also shown with broken lines. 
too large (flooding in $W_{F}$ ). The predicted results are still in good agreements with the experimental results which have large scatter (Peng et al., 2015). The deviation might result from the assumptions like the flooded region for this study, depicted in Fig. 7(b) and the difference of contact angles compared to experiments and so on. The shaded areas mark flooding occurring at $W_{D}=600,218$, and $127 \mu \mathrm{m}$ for subcooling of 2, 4, and $6 \mathrm{~K}$, respectively. The dashed line in Fig. 12(d) implies no flooding (diminishing subcooling).

\subsection{Optimized size effects for 1-D biphilic surface}

To demonstrate the optimal performance, we choose a subcooling of 2 $\mathrm{K}$, and examine the variations of the average heat transfer coefficient with respect to the $W_{F}$ for several $W_{D}$ from 100 to $5000 \mu \mathrm{m}$, as shown in Fig. 13. The end points for all the lines are the onset of flooding given by Fig. 11. The loci of the end points to all curves on the top left portion of the figure representing the maximum heat transfer coefficient are marked by the red dashed line. There are two regimes, the biphilic size effect regime, and the biphilic no size effect regime, and these are consistent with the results of Fig. 6 (for the DWC domain only). The two regimes are separated by the hydrophobic strip width of $2000 \mu \mathrm{m}$. The dotted line shows the upper limit for the hydrophobic surface only, the hydrophobic strip width is in the range of $2 \mathrm{~mm}$ and $200 \mathrm{~mm}$ (no size effect DWC). The marked point on the left axis is the experimental result for the hydrophobic surface only (23\% below the maximum measured 1-D biphilic surface results in (Peng et al., 2015)). The marked point on the top of the right axis is the predicted maximum for a biphilic surface with $W_{D}$ of $200 \mathrm{~mm}$.

We compare three sets of experimental results (Peng et al., 2015) with our predictions. The hollow orange symbols represent the predictions while the filled symbols represent the experiments. The hollow and filled squares overlap, so, for $W_{F}=930 \mu \mathrm{m}$ and $W_{D}=2100$ $\mu \mathrm{m}$, implying a very close match between prediction and experiment. For $W_{F}=450 \mu \mathrm{m}$ and $W_{D}=550 \mu \mathrm{m}$ combination, the prediction is about $16 \%$ higher. For the combination $W_{F}=2100 \mu \mathrm{m}$ and $W_{D}=2500 \mu \mathrm{m}$, the difference is rather large and this can be due to the extent flooding occurs along the hydrophobic strip which is not accounted for in our model, so flooding in the biphilic no size effect regime with large $W_{F}$ and $W_{D}$ needs closer examination.

Since the 1-D biphilic surface enhances DWC in the size effect regime, the variation of the maximum heat transfer coefficient with respect to the $W_{F}$ for $W_{D}$ from 100 to $1200 \mu \mathrm{m}$ and subcooling of 0.1 , $0.5,1,2,4$, and $6 \mathrm{~K}$ is shown in Fig. 14. With decrease of the $W_{D}$, the optimal $W_{F}$ (which is $W_{F, c}$ ) also decreases, the overall heat transfer coefficient increases, reaching a peak (optimal) and then decreases. The optimal $W_{F, c}, W_{D}$ pairs are marked for each subcooling.

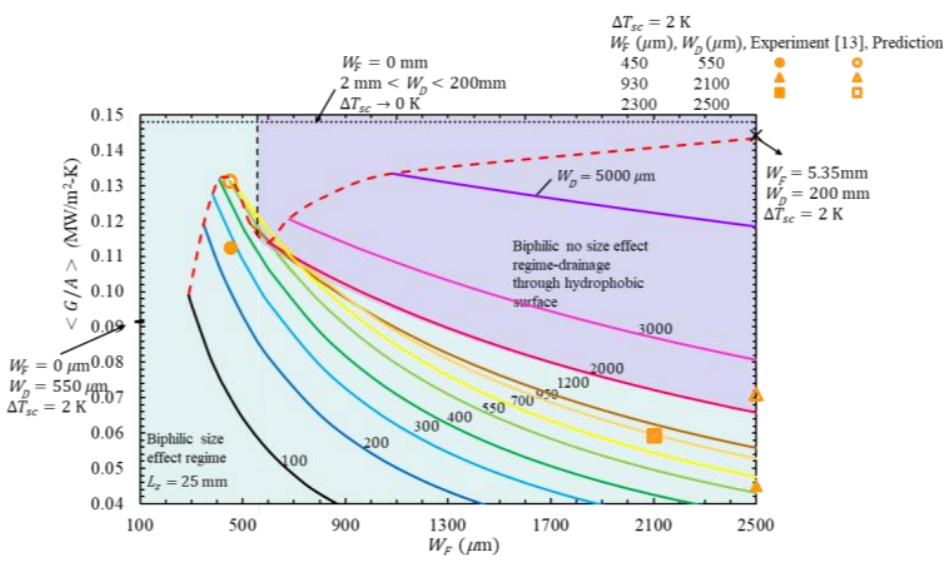

Fig. 13 Variations of the predicted average heat transfer coefficient with respect to the hydrophilic strip width, for a subcooling of $2 \mathrm{~K}$ and different hydrophobic strip widths. Two regimes are identified as size effect (enhancement occurs), and no size effect regimes ( $W_{D}$ itself could generate flooding and decrease $\langle G / A\rangle$ obviously but is not shown here).
With the decrease in subcooling, smaller $W_{D}$ (which follows smaller $W_{F, c}$ ) can be used and the size effect shows in the enhanced average heat transfer coefficient. The optimal $W_{F, c}, W_{D}$ pair become smaller as the subcooling decreases. The broken line connects the loci of the optimal pairs giving the maximum average heat transfer coefficient for different subcooling values. For subcooling $<0.1 \mathrm{~K}$, the maximum average heat transfer coefficient increases to a high value, although such a small subcooling $0.1 \mathrm{~K}$ is difficult to achieve in practice. The optimal $W_{F, c}, W_{D}$ for $2 \mathrm{~K}$ in experiment (Peng et al., 2015) is $450,550 \mu \mathrm{m}$ while the result from our model is $435,528 \mu \mathrm{m}$ which is really close.

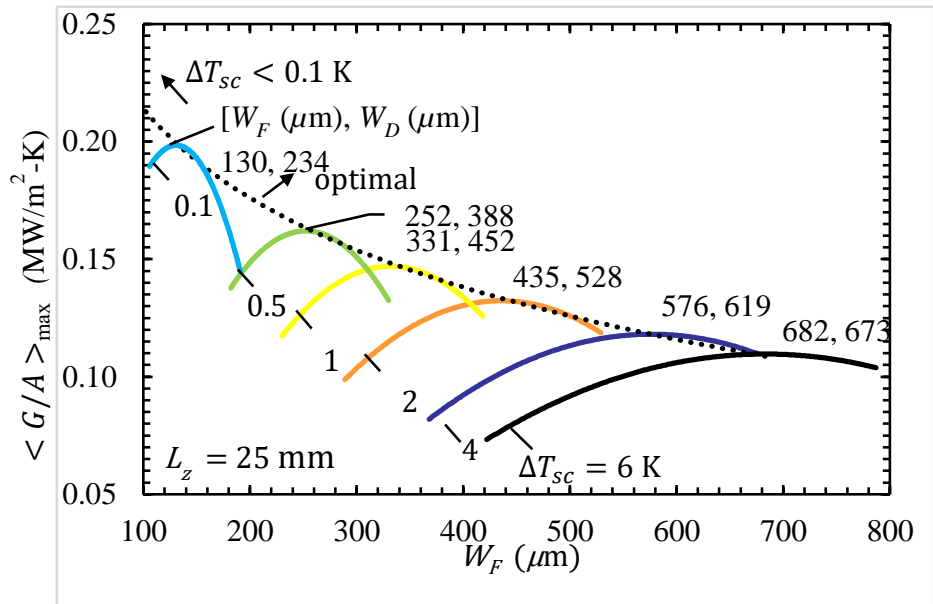

Fig. 14 Variations of the predicted maximum (optimal) heat transfer coefficient with respect to the hydrophilic stripe width, for various subcooling. For each subcooling the effect of variation in the hydrophobic strip width is also shown, along with the values for the peak performance (broken line). The above results were for a circular condenser to compare with the experiments.

To generalize the results, a 1-D rectangular biphilic surface is also considered. The variations of the maximum average heat transfer coefficient with respect to condenser length $L_{z}$ for subcooling of $0.1,0.5$, 1, 2, 4, and $6 \mathrm{~K}$ are shown in Fig. 15(a), (b) for $W_{D}$ of 950 and $600 \mu \mathrm{m}$ respectively. For different subcooling, the maximum heat transfer coefficient starts at different point for a constant length. With the decrease of $L_{z}$, the maximum heat transfer coefficient increases. In the limit of $L_{z} \rightarrow 0$, all the lines tend to converge to the predicted heat transfer coefficient for a single hydrophobic surface by dropwise condensation simulation. The dashed line shows when subcooling is small enough, the heat transfer coefficient becomes independent of the condenser length. For different $W_{D}$, the trends remain the same while the maximum heat transfer coefficient for diminishing length rises for smaller $W_{D}$ due to the size effect in Fig. 6. Figure 15 could directly show this.

\section{SUMMARY AND CONCLUSIONS}

The hydrophobic vertical surfaces cause dropwise condensation allowing for higher heat transfer coefficient (or specific conductance G/A) compared to filmwise condensation on hydrophilic surfaces. The 1-D biphilic surfaces made of periodic hydrophobic and hydrophilic strips allow for efficient draining of the condensate generated from the $W_{D}$ through the $W_{F}$ and can further enhance surface averaged $G / A$, i.e., $\langle G / A\rangle$. In addition, for small $W_{D}$, the droplets adjacent to the $W_{F}$ merge with the draining liquid rivulet there before reaching their maximum departing radii and this causes enhancement in the $G / A$ within $W_{D}$ (called size effect of DWC). Also noting that the $G / A$ within $W_{F}$ is negligible due to the rather thick flowing liquid rivulet there. Additionally, the rivulet can become unstable and cause flooding when the condensate flow rate reaches a critical value. This threshold is reached when the viscous pressure drop along the rivulet (along the direction for gravity) reaches 
the capillary pressure within it. The viscous pressure drop depends on the mass flux and the geometry of liquid rivulet while the capillary pressure is determined by the contact angle $\theta_{c}$ and $W_{F}$. Then the 1-D biphilic surface can be optimized to maximize the size effect of $W_{D}$ while keeping $W_{F}$ to a minimum and avoiding flooding.

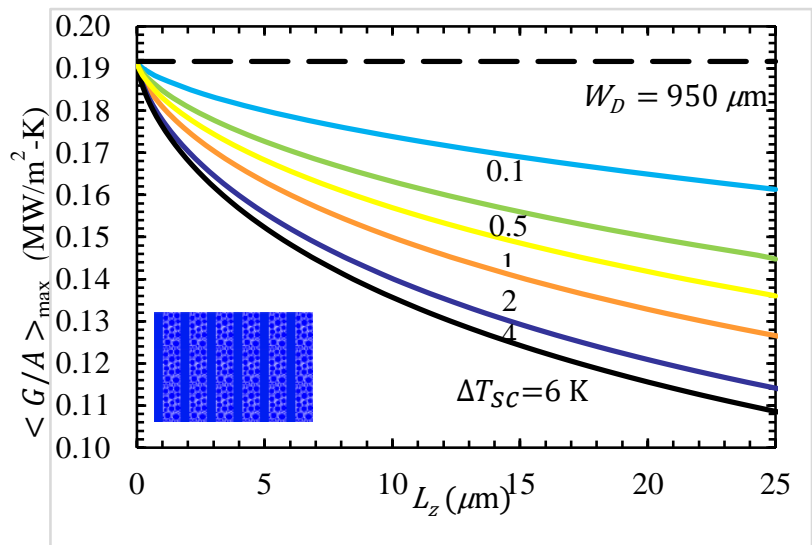

(a)

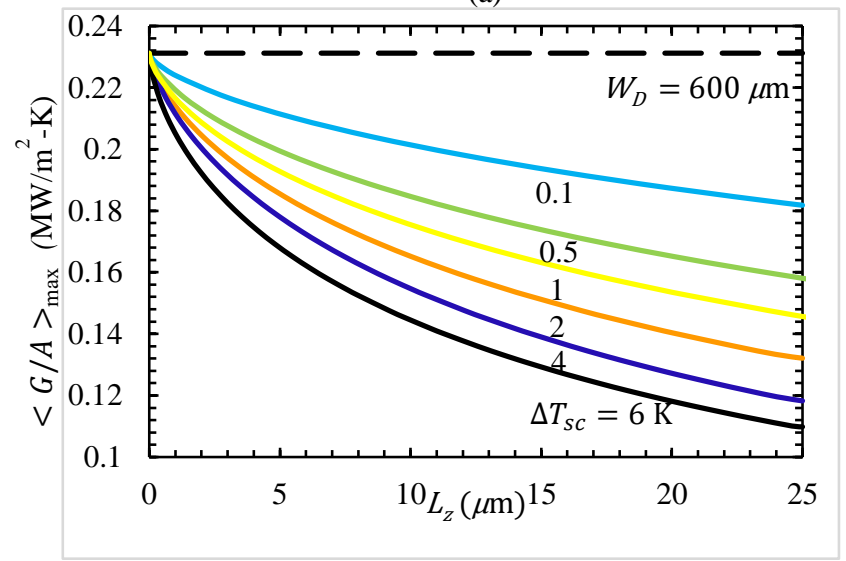

(b)

Fig. 15 Variations of the maximum heat transfer coefficient with respect to the condenser length, for several subcooling. (a) $W_{D}=950$, and (b) $600 \mu \mathrm{m}$. The results are for a 1-D rectangular biphilic surface.

Here through direct simulations of the droplet nucleation, growth, coalescence, merging at the boundaries of $W_{D}$, re-nucleation and departure and through the direct simulations of the rivulet condensate flow and pressure drop within $W_{F}$, the area average $\langle G / A\rangle$ for the total 1-D biphilic surface is predicted for condensation and contact angles of $90^{\circ}$ and $60^{\circ}$ for $W_{D}$ and $W_{F}$ are used respectively. The results are summarized below.

(i) Figure 4 shows a snapshot for direct DWC simulation and the video of the droplet nucleation, growth, coalescence, merging at the boundaries of $W_{D}$, re-nucleation and departure is also available.

(ii) The predicted homogeneous hydrophobic surface size effect is shown in Fig. 6, where the size effect begins when $W_{D}$ is smaller than 2 $\mathrm{mm}$ and the $G / A$ increases smoothly till the physical limit of the nucleation site diameter is reached. The results for the lower droplet nucleation density of $10^{7} 1 / \mathrm{cm}^{2}$ rather better match the experimental results, so this is chosen for the rest of the analysis.

(iii) To compare the predicted results with the available experimental results of (Peng et al., 2015), their circular condenser surface and similar conditions are used and shown in Fig. 7, and it is assumed that once the rivulets flood in $W_{F}$, the entire surface below the flooding length $L_{f}$ is covered with the condensate and heat transfer for this ineffective area is negligible. The simulated rivulet profile and the predicted $L_{f}$ for specific geometry are shown in Fig. 8 and Fig. 9. The effect of subcooling on the critical flooding width and length is shown in
Fig. 10. The corresponding minimum $W_{F}$ for no flooding, i.e., $W_{F, c}$ is shown in Fig. 11.

(iv) The predicted size effects (enhancement in $W_{D}$ and flooding in $\left.W_{F}\right)$ on the $\langle G / A\rangle$ under experimental conditions of (Peng et al., 2015) are shown in Fig. 12, and it is shown that under large subcooling the flooding is more pronounced and there are optimal $W_{D}$ and $W_{F}$ values for each subcooling. There are good agreements with the experimental results.

(v) Beyond the conditions of the experiments (Peng et al., 2015), the results are generalized for very small $\left(\Delta T_{s c} \rightarrow 0\right)$ and moderate subcooling, and variable condenser length (a 1-D rectangular biphilic condenser) including very small values (tending to zero). For $\Delta T_{s c}=2 \mathrm{~K}$, the optimal $W_{D}$ and $W_{F}$ values are found and two regimes (the size effect regime where $W_{D}$ is below $2 \mathrm{~mm}$ and the no size effect regime.) are identified which is consistent with Fig. 6 for homogeneous hydrophobic surface. In Fig. 13, we also show that the optimal $\langle G / A\rangle$ has an upper bound which is for $\left(\Delta T_{s c} \rightarrow 0\right)$ with $W_{F}=0$ (i.e., homogeneous hydrophobic surface).

(vi) We also show the effect of $\Delta T_{s c}$ on the optimal $W_{D}$ and $W_{F, c}$ values in Fig. 14, which shows $\langle G / A\rangle_{\max }$ decreasing monotonically with increase in $\Delta T_{s c}$ for the optimal pairs of $W_{D}$ and $W_{F, c}$. Finally, similar monotonic decrease is shown with increasing condenser length in Fig. 15 .

The predicted results show that for moderate $\Delta T_{s c}$ and condenser length ranges, optimal $W_{D}$ and $W_{F}$ values give similar enhanced $\langle G / A\rangle$ results reported in several experiments. Therefore, with a proper combination of the $W_{F}$, the $W_{D}$, the $L_{z}$ and $\Delta T_{s c}$, we can get a better enhancement of overall heat transfer coefficient in 1-D biphilic surface.

\section{NOMENCLATURE}

$A R \quad$ specific thermal resistance $\left[\mathrm{K} /\left(\mathrm{W} / \mathrm{m}^{2}\right)\right]$

$c_{k} \quad$ film-thickness to droplet-radius ratio

$D \quad$ distance between two droplets (m)

$g \quad$ gravity $\left(\mathrm{m} / \mathrm{s}^{2}\right)$

$G \quad$ Conductance (W/K)

$h \quad$ heat transfer coefficient $\left[\mathrm{W} /\left(\mathrm{m}^{2}-\mathrm{K}\right)\right]$

$\Delta h_{l g} \quad$ heat of vaporization $(\mathrm{J} / \mathrm{kg})$

$k \quad$ thermal conductivity $(\mathrm{W} / \mathrm{m}-\mathrm{K})$

$L \quad$ length $(\mathrm{m})$

$\dot{m} \quad$ mass flux $\left(\mathrm{kg} / \mathrm{m}^{2}-\mathrm{s}\right)$

$n_{0} \quad$ droplet nucleation density $\left(1 / \mathrm{cm}^{2}\right)$

$n_{d} \quad$ number of droplets

$p \quad$ pressure $(\mathrm{Pa})$

$\Delta p \quad$ pressure drop $(\mathrm{Pa})$

$q \quad$ heat flux $\left(\mathrm{W} / \mathrm{m}^{2}\right)$

$Q \quad$ heat flow rate $(\mathrm{W})$

$r \quad$ radius $(\mathrm{m})$

$t \quad$ time (s)

$T \quad$ temperature $\left({ }^{\circ} \mathrm{C}\right.$ or $\left.\mathrm{K}\right)$

$\Delta T \quad$ temperature difference $\left({ }^{\circ} \mathrm{C}\right.$ or $\left.\mathrm{K}\right)$

$u \quad$ velocity $(\mathrm{m} / \mathrm{s})$

$V \quad$ volume $\left(\mathrm{m}^{3}\right)$

$W \quad$ width (m)

$X \quad$ droplet coverage fraction

$x, y, z \quad$ coordinate $(\mathrm{m})$

\section{Greek Symbols}

$\alpha \quad$ area fraction

$\delta \quad$ film thickness (m)

$\rho$ density $\left(\mathrm{kg} / \mathrm{m}^{3}\right)$

$\theta_{c} \quad$ contact angle $\left({ }^{\circ}\right)$

$\mu \quad$ viscosity (N-s/ $\left.\mathrm{m}^{2}\right)$

$\sigma \quad$ surface tension $(\mathrm{N} / \mathrm{m})$ 


$\begin{array}{ll}\text { Subscripts } & \\ b & \text { big droplet } \\ c & \text { critical value } \\ \text { con } & \text { constriction } \\ d & \text { droplet } \\ D & \text { dropwise condensation } \\ f & \text { flooding } \\ F & \text { filmwise condensation } \\ g & \text { gas or vapor } \\ i & \text { initial condition } \\ \text { inter } & \text { interfacial } \\ l & \text { liquid } \\ l g & \text { liquid-vapor saturation } \\ \text { max } & \text { maximum } \\ p & \text { patch method } \\ s & \text { small droplet } \\ s c & \text { subcooling } \\ & \\ \text { others } & \\ <> & \text { area averaged }\end{array}$

\section{REFERENCES}

Alwazzan, M., Egab, K., Peng, B., Khan, J., and Li, C., 2017, "Condensation on hybrid-patterned copper tubes (I): Characterization of condensation heat transfer," International Journal of Heat and Mass Transfer, 112, 991-1004.

https://dx.doi.org/10.1016/j.ijheatmasstransfer.2017.05.039

Barati, S.B., Pinoli, J.-C., Gavet, Y., and Valette, S., 2017, "Differential and average approaches to Rose and Mei dropwise condensation models," International Journal of Mathematical Models and Methods in Applied Sciences, 11, 40-46.

Bostwick, J., and Steen, P., 2018, "Static rivulet instabilities: Varicose and sinuous modes," Journal of Fluid Mechanics, 837, 819-838.

https://dx.doi.org/10.1017/jfm.2017.876

Carey, V.P., 1992, Liquid-vapor Phase-Change Phenomena, Hemisphere Publishing Corporation.

Chen, X., Lu, J., and Tryggvason, G., 2019, "Numerical simulation of self-propelled non-equal sized droplets," Physics of Fluids, 31, 052107 https://dx.doi.org/10.1063/1.5094757

Dietza, C., Rykaczewski, K., Fedorov, A. G., and Joshi, Y., 2010, "Visualization of droplet departure on a superhydrophobic surface and implications to heat transfer enhancement during dropwise condensation," Applied Physics Letters, 97, 033104.

https://dx.doi.org/10.1063/1.3460275

Ghosh, A., Beaini, S., Zhang, B. J., Ganguly, R., and M. Megaridis, C., 2014, "Enhancing Dropwise Condensation through Bioinspired Wettability Patterning", Langmuir, 30(43), 13103-13115. https://dx.doi.org/10.1021/la5028866

Glicksman, L.R., and Hunt JR, A.W., 1972, "Numerical simulation of dropwise condensation," International Journal of Heat and Mass Transfer, 15, 2251-2269. https://dx.doi.org/10.1016/0017-9310(72)90046-4

Hassan, G., Yilbas, B., Al-Sharafi, A. and Al-Qahtani, H., 2019, "selfcleaning of a hydrophobic surface by a rolling water droplet," Scientific Reports, 9, 5744.

https://dx.doi.org/10.1038/s41598-019-42318-3
Hoenig, S.H., and Bonner, R.W., 2018, "Dropwise Condensation on Superhydrophobic Microporous Wick Structures," Journal of Heat Transfer, 140, 071501-1. https://dx.doi.org/10.1115/1.4038854

Hu, H., and N. Chung, J., 2018, "A molecular dynamics simulation of nanoscale water vapor absorption on the surface of $\mathrm{LiBr}$ aqueous solution," Frontiers in Heat and Mass Transfer, 11, 24.

https://dx.doi.org/10.5098/hmt.11.24

Kim, M.-K., Cha, H., Birbarah, P., Chavan, S., Zhong, C., Xu, Y., and Miljkovic, N., 2015, "Enhanced Jumping-Droplet Departure," Langmuir, 31(49), 13452-13466 https://dx.doi.org/10.1021/acs.langmuir.5b03778

Leach, R.N., Stevens, F., Langford, S.C., and Dickinson, J.T., 2006, "Dropwise Condensation: Experiments and Simulations of Nucleation and Growth of Water Drops in a Cooling System," Langmuir, 22(21), 8864-8872.

https://dx.doi.org/10.1021/la061901+

Le Fevfie, E.J., and Rose, J.W., 1966, "A theory of heat transfer by dropwise condensation," Proc. Third Int. Heat Transfer Conference, Am. Inst. Chem. Engrs. New York 2,362.

Lu, C.-H., Beckmann, M., Unz, S., Gloess, D., Frach, P., Holst, E., Lasagni, A., and Bieda, M., 2015, "Heat transfer model of dropwise condensation and experimental validation for surface with coating and groove at low pressure", International Journal of Heat and Mass Transfer, 52(1), 113-126.

https://dx.doi.org/10.1007/s00231-015-1641-0

Mikic, B., 1969, "On mechanism of dropwise condensation," International Journal of Heat and Mass Transfer, 12(10), 1311-1323. https://dx.doi.org/10.1016/0017-9310(69)90174-4

Modak, S., Kaviany, M., Hoeing, S.H., and Bonner, R.W., 2019, "Numerical analysis of meniscus dynamics in monolayer-wick dropwise condensation," J. Numerical Heat Transfer, 76(5), 301-322. https://dx.doi.org/10.1080/10407782.2019.1627829

O’Neill, G. A., and Westwater, J.W., 1984, "Dropwise condensation of steam on electroplated silver surfaces," International Journal of Heat and Mass Transfer, 27(9), 1539-1549.

https://dx.doi.org/10.1016/0017-9310(84)90266-7

Peng, B., Ma, X., Lan, Z., Xu, W., and Wen, R., 2015, "Experimental investigation on steam condensation heat transfer enhancement with vertically patterned hydrophobic-hydrophilic hybrid surfaces," International Journal of Heat and Mass Transfer, 83, 27-38. https://dx.doi.org/10.1016/j.ijheatmasstransfer.2014.11.069

Rose, J.W., 1966, "On the mechanism of dropwise," International Journal of Heat and Mass Transfer, 10, 755-762. https://dx.doi.org/10.1016/0017-9310(67)90135-4

Rose, J.W., 2002, "Dropwise condensation theory and experiment: a review", Proceedings of the Institution of Mechanical Engineers, Part A: Journal of Power and Energy, 216(2), 115-128. https://dx.doi.org/10.1243/09576500260049034

Rose, J.W. and Glicksman, L.R., 1972, "Dropwise condensation-The distribution of drop sizes," International Journal of Heat and Mass Transfer, 16, 411-425.

https://dx.doi.org/10.1016/0017-9310(73)90068-9 
Shang, Y., Hou, Y., Yu, M., and Yao, S., 2018, "Modeling and optimization of condensation heat transfer at biphilic interface," International Journal of Heat and Mass Transfer ,122, 117-127. https://dx.doi.org/10.1016/j.ijheatmasstransfer.2018.01.108

Tsuruta, T., and Tanaka, H., 1991, "A theoretical study on the constriction resistance in dropwise condensation," International Journal of Heat and Mass Transfer, 34(11), 2779-2786.

https://dx.doi.org/10.1016/0017-9310(91)90237-9

Van Dyke, A.S., Collard, D., M Derby, M., and Rachel Betz, A., 2015 "Droplet coalescence and freezing on hydrophilic, hydrophobic, and biphilic surfaces," Applied Physics Letters, 107, 141602. https://dx.doi.org/10.1063/1.4932050

Wilkins, D. G., Bromley, L. A. and Read, S. M., 1973, "Dropwise and filmwise condensation of water vapor on gold,"

AIChE Journal, 19(1), 119-123.

https://dx.doi.org/10.1002/aic.690190117
Wu, Y., Yang, C., Yuan, X., 2001, "Drop distributions and numerical simulation of dropwise condensation heat transfer," International Journal of Heat and Mass Transfer, 44(23), 4455-4464. https://dx.doi.org/10.1016/S0017-9310(01)00085-0

Yunus, M., and S. Alsoufi, M., 2019, "Development of a heat pipe and grey based Taguchi method for multi-output optimization to improve thermal performance using hybrid nanofluids," Frontiers in Heat and Mass Transfer, 12, 11.

https://dx.doi.org/10.5098/hmt.12.11

Zarei, S., Talesh Bahrami, H.R., and Saffari, H., 2018, "Effects of geometry and dimension of micro/nano-structures on the heat transfer in dropwise condensation: A theoretical study," Applied Thermal Engineering, 137, 440-450.

https://dx.doi.org/10.1016/j.applthermaleng.2018.04.003 